\title{
INFESTATION EXPÉRIMENTALE \\ PAR LES LARVES PLÉROCERCOIDES DE \\ DIPHYLLOBOTHRIUM MANSONI AU TONKIN
}

\author{
Par H. GALLIARD
}

Les recherches que nous avons effectuées étaient destinées à étudier les voies de pénétration des Sparganum chez des batraciens et des mammifères, ainsi que les migrations effectuées dans l'organisme de ces hôtes et les lésions déterminées par leur passage. Elles ont porté sur le batracien le plus communément trouvé au Tonkin, Rana tigrina, sur le cobaye, le rat, le lapin, le chien et le chat.

Rana tigrina. - Les expériences ont été faites en tenant compte du nombre de têtes ingérées, de la durée de l'expérience et du nombre de têtes retrouvées. L'infestation a eu lieu dans tous les cas par voie buccale.

Les Sparganum provenaient de Rana tigrina, de $R$. limnocharis et de Tropidonotus piscator.

On voit que l'infestation réussit dans presque tous les cas. En outre, la voie de pénétration est toujours l'estomac (1). Le nombre de têtes retrouvées semble être proportionnellement. plus élevé quand la quantité ingérée est plus importante. Avec une seule tête nous n'avons pas réussi. Ce fait est peut-être dû, dans ce cas, à ce que, isolées, elles sont digérées, alors que les sucs digestifs sont insuffisants pour agir sur un nombre plus grand. C'est très net dans le cas II où le nombre des têtes retrouvées est identique à celui des têtes absorbées. Il ne peut pas y avoir de confusion avec une infestation ancienne, car, dans ce cas, particulier, il n'y avait aucune larve dans les muscles.

La traversée de la paroi gastrique est en général rapide. Elle débute dès la pénétration de la larve dans la cavité, et elle s'effectue en six heures.

(1) Joyeux et Baer (1929) ont montré qu'il en est de même pour $S$. ranarum quand l'infestation se fait par voie buccale ; mais, introduits par voie rectale, les Sparganum pénètrent par l'intestin.

Ann. de Parasitologe, T. XXIII, Nos $3-4$. - 1948, p. 203-213. 


\begin{tabular}{|c|c|c|c|c|}
\hline & $\begin{array}{c}\text { NOMBRE } \\
\text { DE } \\
\text { Scolex }\end{array}$ & $\begin{array}{l}\text { DURÉE } \\
\text { DE } \\
\text { l'expér. }\end{array}$ & $\begin{array}{l}\text { NOMBRE } \\
\text { retrouvé. }\end{array}$ & Localisation \\
\hline I. . & 24 & $5 \mathrm{j}$. & 18 & $\begin{array}{l}\text { Ds. paroi gastrique, sur œsophage, ds. } \\
\text { mésentères, muscles (aussi longs que } \\
\text { ceux ingérés). }\end{array}$ \\
\hline II $\ldots \ldots$. & 19 & $23 \mathrm{j}$. & 19 & $\begin{array}{l}\text { Paroi estomac, surface foie, corps adi- } \\
\text { peux, mésogastrique, rien dans les } \\
\text { muscles. }\end{array}$ \\
\hline III . . . . . & 17 & $6 \mathrm{j}$. & 10 & $\begin{array}{l}\text { 2, paroi ext. estomac sous péritoine } \\
3 \text {, mésentère } ; 1 \text {, foie. }\end{array}$ \\
\hline IV ....... & 17 & $7 \mathrm{j}$. & 16 & Environs estomac, paroi abd. antér. \\
\hline$V \ldots \ldots$ & 12 & $6 \mathrm{~h}$. & 3 & Sortant de paroi gastrique, près cardia. \\
\hline VI....... & 10 & $3 \mathrm{j}$. & 3 & $\begin{array}{l}\text { 1, ds. paroi estomac ; } 2 \text {, travers, paroi } \\
\text { abdominale. }\end{array}$ \\
\hline VII. & 10 & $24 \mathrm{~h}$. & 3 & Adhérent à face externe de l'estomac. \\
\hline VIII ..... & 9 & $4 \mathrm{j}$. & 7 & $\begin{array}{l}\text { 3, cavité péritonéale ; } 2 \text {, paroi abd. } \\
\text { antér. ; } 1 \text {, rétro-œsoph. ; près globe } \\
\text { oculaire gauche, sur trajet nerf opti- } \\
\text { que. Rien dans muscles. }\end{array}$ \\
\hline IX... & 6 & $4 \mathrm{j}$. & 4 & Traversant paroi abdominale. \\
\hline & 6 & $2 \mathrm{~h}$. & 2 & Dans paroi gastrique. \\
\hline XI...... & 5 & $15 \mathrm{j}$. & 0 & \\
\hline XII ...... & 5 & $8 \mathrm{~h}$. & 2 & Dans paroi gastrique \\
\hline XIII..... & 5 & $4 \mathrm{~h}$. & 4 & $\begin{array}{l}\text { 2, dans paroi gastrique } ; 2 \text {, fixés sur } \mathrm{f} \text {. } \\
\text { ext. de paroi. }\end{array}$ \\
\hline XIV. & 5 & $8 \mathrm{~h}$. & 3 & Surface externe de l'estomac. \\
\hline XV... & 1 & $8 \mathrm{~h}$. & 0 & \\
\hline XVI. & 1 & $4 \mathrm{~h}$. & 0 & \\
\hline
\end{tabular}

Mais le passage peut se faire de deux facons. Ou bien directement (fig. 1), en embrochant toute l'épaisseur de la paroi, ou bien en deux temps. La larve pénètre dans la paroi et se loge d'abord entièrement entre la muqueuse et la musculeuse avant de poursuivre sa migration (fig. 2). Elle s'y trouve déjà, entière, au bout de deux heures et y reste 24 à 48 heures, parfois jusqu'à 4 ou 5 jours. De 8 heures à 6 jours, les têtes peuvent être trouvées sur la surface externe de la paroi, sous le péritoine. Elles peuvent y rester une vingtaine de jours. Dès le $5^{\circ}$ jour, on trouve des larves sur la paroi externe de l'œesophage, dans la cavité péritonéale, dans le mésogastrique, à la surfacé du foie ou sous la capsule de Glisson, dans le corps adipeux, dans la tête, sur le trajet du nerf optique. 
La durée de la migration des larves de l'estomac aux muscles est difficile à préciser (1). Elle est probablement variable. Au bout de 3 et 4 jours, nous avons vu des larves passant du péritoine aux muscles abdominaux. Mais dans un autre cas (II) elles étaient encore, le

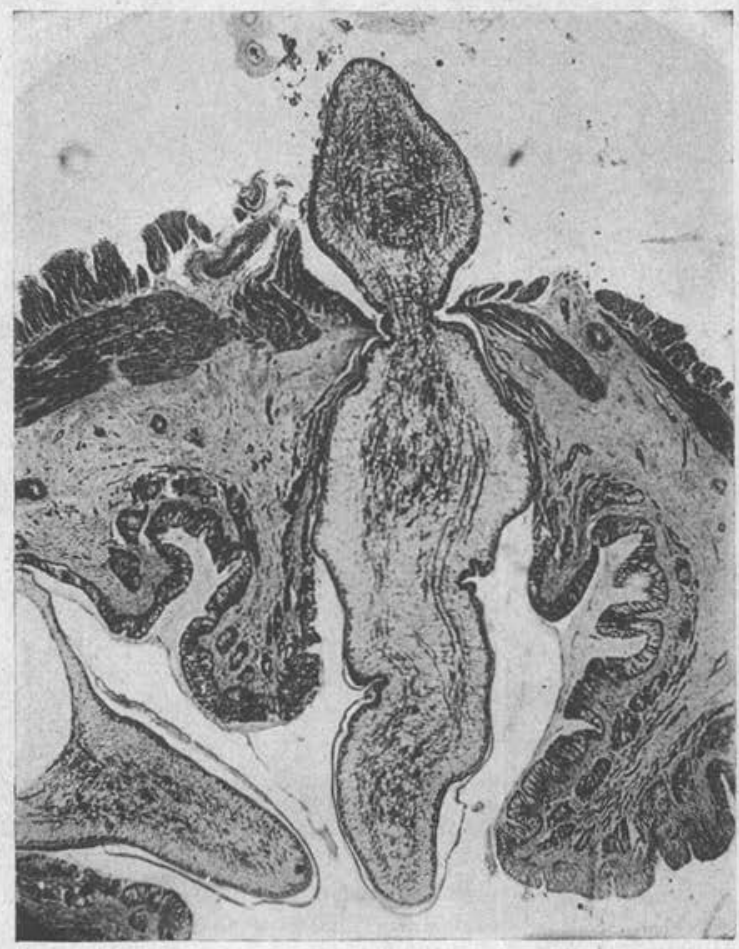

Fig. 1. - Sparganum mansoni traversant directement la paroi gastrique de Rana tigrina.

$23^{\circ}$ jour, autour de l'estomac et dans le mésogastrique, et il n'y en avait aucune dans les muscles. Pour Kobayashi, toutes les larves seraient localisées dans les muscles fémoraux le $32^{\circ}$ jour.

La voie par laquelle elles émigrent vers les muscles fémoraux est variable. Nous avons le plus souvent vu des larves en parties libres

(1) Nos expériences ont été faites à la température de 25-28\%. Il est bien évident que la durée de la migration à travers la paroi digestive dépend de la température extérieure. D’après Nagoya, chez la grenouille, la cavité péritonéale est atteinte en deux heures en septembre, tandis qu'en février, au bout de 11 heures, le ver n'a encore aucune tendance à quitter la cavité gastrique. 
dans la cavité péritonéale, pénétrant à travers la paroi abdominale antérieure dans le tissu sous-cutané. Kobayashi (1931) (1) admet aussi qu'elles peuvent passer de la cavité périrectale directement dans les muscles de la cuisse.

治

En ce qui concerne les dimensions des Sparganum, nous avons confirmé les faits observés par Joyeux et Baer (1931) et par Muel-

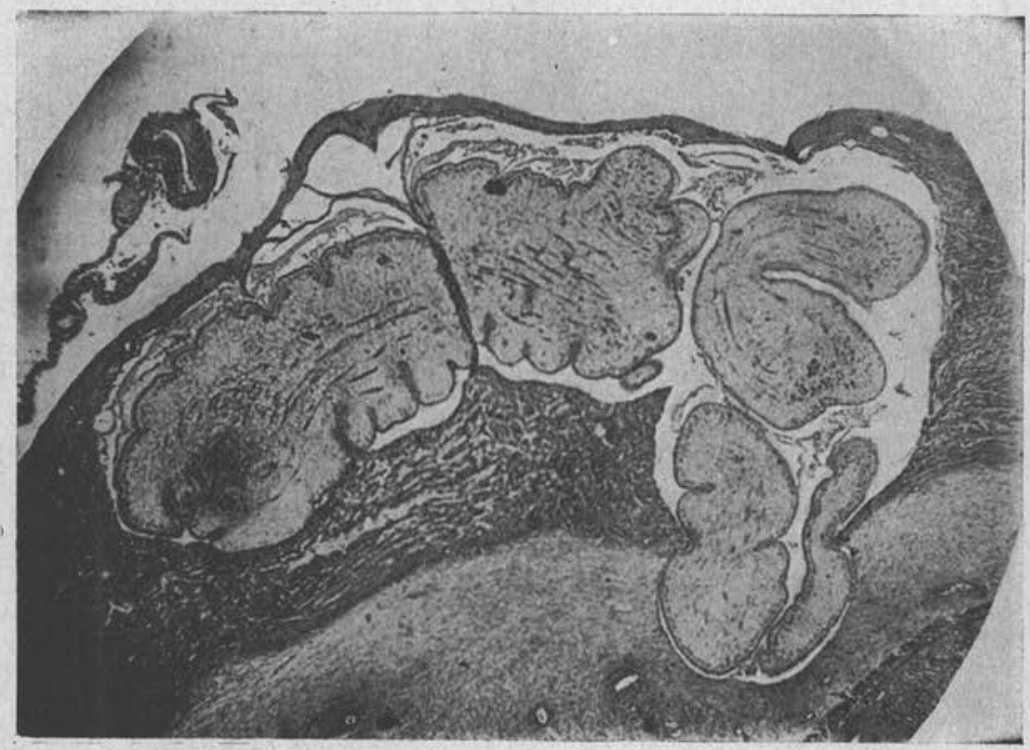

Fig. 2. - Sparganum mansoni sortant de la sous-muqueuse pour se loger dans la couche musculaire gastrique de Rana tigrina.

ler (1938) sur la scission de la tête avant la traversée de la paroi gastrique. Notons aussi que les meilleurs résultats obtenus (cas IV) l'ont été avec des larves sectionnées, aussi près que possible de la tête (1 à $2 \mathrm{~mm}$.), ce qui a probablement facilité et hâté leur pénétration.

Au point de vue histologique, on peut constater que chez la gre-

(1) D'après Kobayashi, quelques plérocercoïdes, après passage à travers la paroi digestive, pénètrent aussi par le diaphragme dans la cavité thoracique, puis dans la paroi. Leur tendance à émigrer vers les muscles fémoraux est contraire à ce qui se passe chez la souris où la migration se fait en avant vers les muscles du cou. D'après Nagoya, de la cavité péritonéale, les larves parviennent au corps thyroïde et au coeur et pénètrent dans les muscles. Pour Mueller, chez le "leopard frog », le Sparganum de D. mansonoides semble avoir une prédilection pour le foie. 
nouille, il n'y a jamais de lésions apparentes dans aucun organe du fait du passage ou de la présence d'un Sparganum. Même quand il y a passage direct à travers la paroi gastrique, il n'est jamais possible d'en retrouver la trace. Il ne se produit aucune réaction tissulaire, ni trace d'inflammation au passage du ver (fig. 1). Les tissus sont lysés, dissociés, au fur et à mesure, à son contact. Il en est de même, lorsqu'il est logé dans l'épaisseur de la paroi. Il y a adaptation parfaite entre le parasite et l'hôte.

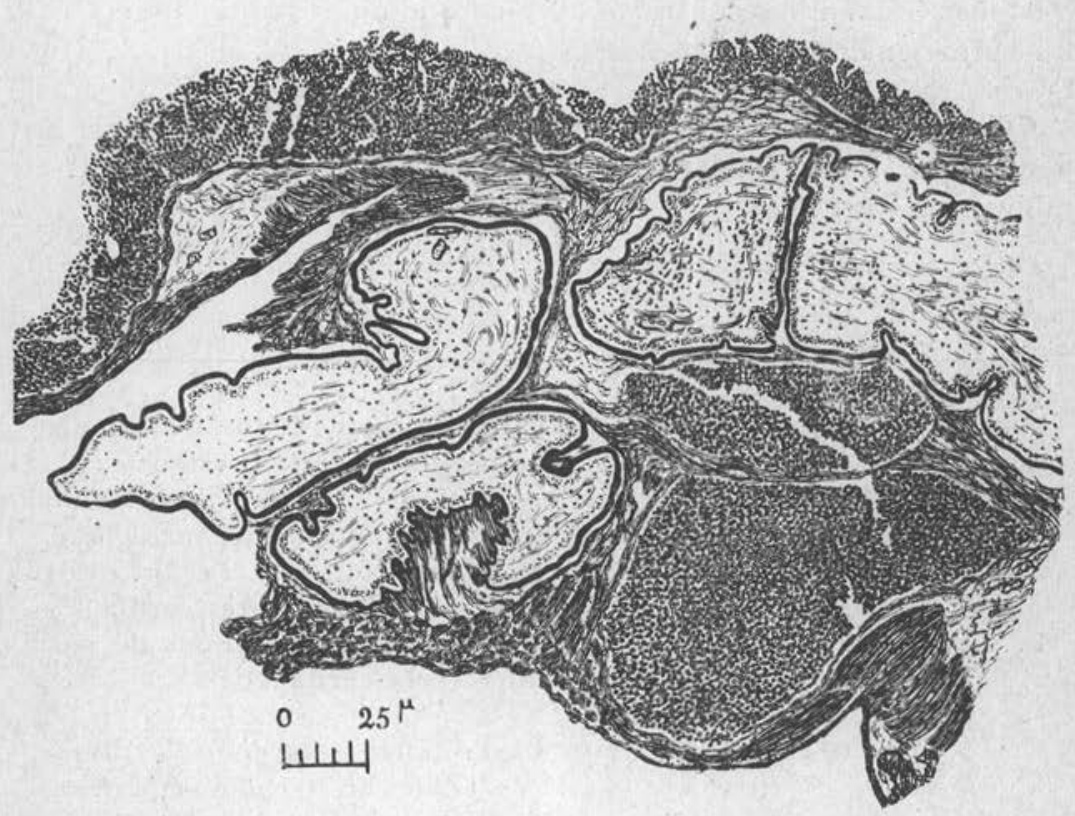

Fig. 3. - Sparganum mansoni faisant effraction de la sous-muqueuse gastrique dans la cavité péritonéale du cobaye.

Autres batraciens et reptiles. - L'infestation de R. tigrina a pu se faire avec des Sparganum de $R$. limnocharis. Inversement, nous avons pu obtenir la migration à travers la paroi gastrique de $R$. limnocharis avec des larves de $R$. tigrina. De même, $R$. tigrina est réceptive aux larves de Tropidonotus piscator. $T$. piscator est réceptif, mais il est si souvent et si fortement infesté, qu'il est difficile de présicer les localisations récentes.

Par contre, nous avons signalé par ailleurs (1946) que 
Hypsirrhina enhydris n'avait jamais été trouvée parasitée. Ainsi, nous avons tenté l'infestation d'un spécimen provenant d'un lot de 13 exemplaires indemnes. Sur 8 Sparganum de $R$. limnocharis ingérés, 5 ont été retrouvés le $5^{\circ}$ jour : un sur la paroi externe de l'estomac, un sur la paroi de l'œesophage, un dans les muscles du cou, un dans les muscles de l'extrémité postérieure. Ils mesuraient tous $1 \mathrm{~cm}$. à $1 \mathrm{~cm}$. 5 , ce qui est en faveur de leur localisation récente, puisqu'au moment de l'ingestion ils avaient 4 à $7 \mathrm{~cm}$. de longueur. Si c'est exact, cette pénétration rapide dans les muscles chez les reptiles contraste avec ce que nous avons observé chez les batraciens.

Cobaye. - Nos essais de réencapsulement de Sparganum de grenouille ont réussi également chez le cobaye, comme le montre le tableau suivant :

\begin{tabular}{|c|c|c|c|}
\hline NOMBRE & DURÉE & $\begin{array}{l}\text { NoMirRe } \\
\text { RETrouvé }\end{array}$ & Localisation \\
\hline & $14 \mathrm{~h}$. & 3 & 2. paroi gastrique $; 1$, surface estomac \\
\hline 11. & $19 \mathrm{~h}$. & 1 & Sous péritoine, sortant estomac. \\
\hline & $18 \mathrm{~h}$. & 1 & Ds. paroi près cardia. Ulcér. de mu- \\
\hline & $18 \mathrm{~h}$. & 1 & $\begin{array}{l}\text { Sortant de paroi gastr. Perfor. ovalai- } \\
\text { re de paroi antér. près cardia. }\end{array}$ \\
\hline & $24 \mathrm{~h}$. & 2 & $\begin{array}{l}\text { Ss. pér. gast. ; } 2 \text { ulcérations de paroi } \\
\text { antér. près cardia. }\end{array}$ \\
\hline & $10 \mathrm{~h}$. & 0 & \\
\hline 20 & 60 jrs. & 13 & $\begin{array}{l}\text { 6, libres ds. région sterno-péricardique; } \\
5 \text { ds. adhér. du sternum au péricarde } \\
\text { et poum. gauche ; } 1 \text {, ds. grd. épiploon. } \\
\text { adhérent à par. abd. antér.; I, ds. } \\
\text { adhér. entre foie et estom. }\end{array}$ \\
\hline
\end{tabular}

On voit que la traversée est également rapide, mais moins que chez les grenouilles. Vers la $18^{\circ}$ ou $19^{\circ}$ heure, les larves sortent de la paroi. Parfois, elles sont sur la surface externe dès la $14^{\circ}$ heure. Il n'y a pas de traversée directe.

Contrairement à la grenouille, le cobaye réagit avec violence à la migration des larves : Il se produit des ulcérations et des suffusions hémorragiques. Les perforations persistent dans certains cas.

L'examen histologique traduit ces perturbations. Le ver pro- 
voque une réaction importante à son voisinage : congestion intense, foyers hémorragiques étendus, infiltration massive par les leucocytes mononucléaires et surtout polynucléaires qui forment des microabcès en certains points de la sous-muqueuse, éosinophilie abondante, nécrose de la muqueuse.

Lorsque l'infestation est ancienne (60 jours), les larves restent au contact des viscères et produisent des réactions inflammatoires et des adhérences à l'intérieur desquelles elles restent logées et vivantes : adhérences sterno-péricardiques et sterno-pulmonaires, pariéto-épiploïques, gastro-hépatiques.

Nous n'avons pas observé de localisation intra-musculaire chez le cobaye.

Rat. - Le rat gris, sauvage, est également réceptif.

\begin{tabular}{|c|c|c|c|}
\hline NOMBre & DURÉE & $\begin{array}{l}\text { NoMBre } \\
\text { RETOUVÉ }\end{array}$ & Localisation \\
\hline $5 \ldots \ldots \ldots$ & 24 heures & 2 & $\begin{array}{l}1 \text { ds. pancréas, 1, ds. paroi estomac } \\
\text { (entre muqueuse et musculeuse). }\end{array}$ \\
\hline $6 \ldots \ldots \ldots$ & 24 heures & 1 & Libre dans péritoine. \\
\hline $\begin{array}{c}68 \ldots \ldots . \\
\text { en } 6 \text { fois, } 1 \\
\text { semaine } \\
\text { intervalle, } \\
15 \text { veille } \\
\text { de mort). }\end{array}$ & 36 jours & 25 & $\begin{array}{l}\text { Ds. paroi gastrique, } 2 \text {; sur par. ext., } \\
3 \text {; sur foie et rate, } 10 \text {; cuisses, } 6 \text {; } \\
\text { m. prévertébraux, } 4 \text {. }\end{array}$ \\
\hline
\end{tabular}

La migration est également rapide (1). Elle ne détermine pas cependant de lésions ou de réactions inflammatoires. Dans le troisième cas, la localisation dans les muscles s'est faite sans difficulté, au bout de 36 jours, comme chez la grenouille. La présence de larves dans la paroi gastrique était due à la surinfestation pratiquée la veille de la mort, avec 15 larves.

(1) Il semble que la migration soit beaucoup plus rapide chez le rat et la souris que chez la grenouille ou le cobaye. D'après Nagoya, chez la souris, la cavité péritonéale peut être atteinte en une heure. Mueller a constaté que les Sparganum de $D$. mansonoides sont retrouvés dans la cavité péritonéale de la souris 1 heure 15 après l'ingestion. 
Lapin. - Trois expériences seulement ont été faites avec le lapin.

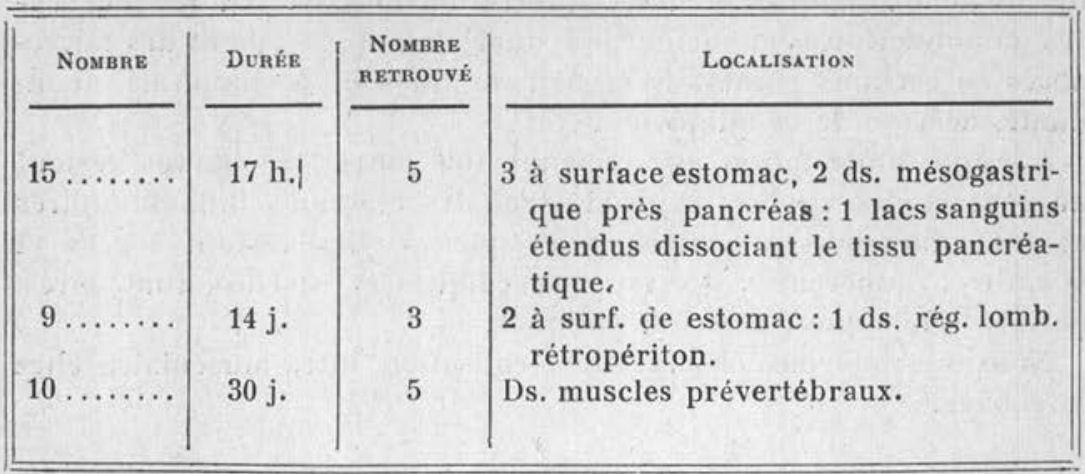

La rapidité de l'évolution est variable : au bout de 14 jours, on trouve encore des larves au voisinage de l'estomac. Les Sparganum ne semblent pas déterminer de réaction inflammatoire au niveau des séreuses. Mais, dans le premier cas, deux larves ont provoqué une hémorragie abondante, dans, le mésogastrique, dissociant le tissu pancréatique. La localisation musculaire a pu être obtenue au bout de 30 jours.

Chien. - L'infestation du chien a été essayée avec des Sparganum de provenance diverse.

1 provenant de l'œil humain. Autopsie au bout de 28 jours : 0 .

1 - $\quad$ - $\quad$ - $\quad$ - $13-0$.

$7 \quad$ - de Rana tigrina. $12^{\circ}$ jour, œufs dans les selles; $17^{\circ}$ jour,

4 bothriocéphales dans l'intestin grêle. 10 - $-\quad-\quad 8^{\circ}$ jour : 1 bothriocéphale dans le grêle. 37 - de têtards de $R$. limnocharis. $30^{\circ}$ jour, œufs dans les selles; $48^{\circ}$ jour, 12 bothrio. de 2 à $8 \mathrm{~cm}$. Anneaux mûrs, $6 \mathrm{~mm}$. de large. 98 de Tropidonotus piscator. Mort 7 mois après, 49 bothrio.

L'infestation réussit done dans tous les cas, sauf avec des Sparganum provenant de la région orbitaire de l'homme. Mais, dans ces cas, l'échec est probablement dû à ce qu'une seule larve a été ingérée. Les précautions habituelles (anthelmintiques, purgatifs, recherche des œufs dans les selles) ont été prises pour éviter les causes d'erreur et s'assurer qu'il s'agissait bien d'une primo-infection. Du reste, sur plusieurs centaines d'autopsie, nous n'avons trouvé que rarement une infestation naturelle. On voit aussi que la surinfestation est possible au bout de 14 jours. 
Chat. - Les résultats ont été les suivants :

10 Sparganum : $30^{\circ}$ jour, œufs dans les selles ; $79^{\circ}$ jour : 5 bothriocéphales de $60 \mathrm{~cm}$. dans le grêle.

20 Sparganum : $28^{e}$ jour : 8 bothriocéphales dans le grêle.

Mêmes remarques qu'en ce qui concerne le chien, à propos de l'infestation naturelle.

Discussion. - Ainsi, le « réencapsulement » de Sparganum mansoni se fait aisément chez les batraciens, les reptiles, les mammifères et surtout le rat gris qui est un hôte naturel au Tonkin comme le signale Houdemer, bien que nous ne l'ayons pas constaté.

Pour Kobayashi (1931), il y aurait, suivant l'animal, d'es différences marquées dans le développement en longueur, qui se ferait plus rapidement chez les animaux à sang chaud. Mais il ne signale pas de différence, en ee qui concerne la possibilité d'infestation.

Joyeux et Baer (1929), avec $S$, ranarum, concluent à l'infestation facile chez les vertébrés à sang froid' et réussissent $\mathbf{2}$ fois chez le hérisson. Ultérieurement, ils obtiennent le réencapsulement chez le furet et le lapin (1 succès : 1 Sparganum dans le mésentère), mais pas chez le rat, le cobaye, la souris. Ultérieurement (1934), ils obtiennent, avec $D$. erinacei, 1 succès avec le rat, la souris, le cobaye, le lapin.

Avec des Sparganum mansoni provenant d'Indochine, ces mêmes auteurs constatent que les résultats sont peu marqués pour tous les groupes y compris les oiseaux. Il n'y a pas de prédilection pour aucun groupe de vertébrés. Un succès fut obtenu avec le rat blanc, mais pas avec le cobaye et le lapin. Mais les auteurs signalent qu'il 's'agissait de Sparganum en mauvais état et qu'aucune conclusion ne peut être tirée.

Chez la grenouille verte, d'après Mueller (1938), S. mansonoides, même s'il arrive à franchir la paroi intestinale, ne conserve pas sa vitalité. Par contre la souris, le rat, certains singes et le « léopard frog sont des hôtes favorables.

L'action pathogène des Sparganum au cours de leurs migrations a fait l'objet de constatations diverses. Nous avons dit que chez les vertébrés à sang froid, nous n'avions jamais constaté de lésions d'aucune sorte. Cependant, d'après Nagoya, le passage des vers dans l'organisme des batraciens provoque de multiples lésions, déchirures ou hémorragies.

Pour les mammifères, le cobaye, le rat et la souris, Tes avis diffè- 
rent. Le cobaye semble particulièrement sensible, d'après notre expérience. Cependant Houdemer, Dodero et Cornet (1933) écrivent que quand l'infestation expérimentale s'est faite par ingestion de Sparganum, on ne constate aucune réaction tissulaire. Ils ont trouvé un Sparganum réencapsulé dans le testicule d'un cobaye sans réaction de voisinage.

Par contre, Feng et Hoeppli ont constaté que le Sparganum de $D$. erinacei produit de larges perforations du tube digestif du hamster chinois, sans d'ailleurs provoquer de péritonite, bien que les larves ou leur émulsion n'aient aucune action bactéricide.

Nagoya a vu que chez la souris les lésions provoquées sont aussi importantes que chez la grenouille, mais ne sont nécrotiques que dans le cas où les larves pénètrent dans les ganglions lymphatiques. Mueller également constate que chez la souris les Sparganum sont parfois entourés d'une paroi de tissu réactionnel et que sur leur passage ils laissent des galeries remplies d'une substance caséeuse, ou des traces fibreuses, ou des restes de tissus nécrosés. Chez le rat, les plérocercoïdes ont la tête entourée d'une zone hémorragique.

\section{RÉSUMÉ}

L'infestation par les larves plérocercoïdes de Diphyllobothrium mansoni est facilement obtenue chez Rana tigrina, $R$. limnocharis, Hypsirrhina enhydris, chez le rat, le cobaye, le lapin, à condition d'utiliser un nombre suffisant de larves. Chez les batraciens et reptiles, le passage et la migration des larves se font sans aucune réaction inflammatoire. Par contre, chez le cobaye, les lésions sont souvent marquées (ulcération, perforation, hémorragie), et les Sparganum provoquent des adhérences péritonéales, pleurales, péricardiques. Le rat semble le plus réceptif. Il ne se produit aucune immunité chez aucun animal, la surinfestation est toujours possible. En outre, les résultats d'infestation croisée entre batraciens et reptiles montrent que le pouvoir infestant des larves n'est pas modifié après séjour chez tel ou tel hôte.

L’infestation du chien a réussi cinq fois avec des Sparganum de Rana tigrina, Tropidonotus piscator, têtards de $R$. limnocharis. Le chat s'infeste aussi facilement.

\section{BIBLIOGRAPHIE}

FeNG (L. C.) et HoEppli (R.). - Sparganum of Diphyllobothrium erinacei as carrier of bacteria and the problem of its bactericidal action. Chin. Med. Jl., L, 1936, p. 1457. 
Galliard (H.) et Ngu (D. V.). - Particularités du développement de Diphyllobothrium mansoni au Tonkin. Ann. de parasit. hum. et comp., XXI, 1946 , p. 246.

Houdemer (E.), Dodero et Cornet. - Les sparganoses animales et la sparganose oculaire en Indochine, Bull. Soc. Méd. Chir. Indochine, 1933, p. 251.

Joyeux (Ch.) et BAER (J.). - Recherches expérimentales sur la larve plérocercoïde de Diphyllobothrium ranarum. C.R. Soc. Biol. Paris, CI, 1929, II, p. 294.

- Sur le réencapsulement de Sparganum ranarum. C.R. Soc. Biol. Paris, CII, 1923 , p. 305.

Evolution des plérocercoïdes de Diphyllobothrium. C.R. Soc. Biol., CVIII, 1931 , p. 97.

Joyeux (Ch.), Houdemer et Baer (J.). - Recherches sur la biologie des Sparganum et l'étiologie de la sparganose oculaire. Bull. Soc. Path. exot., XXVII, 1934 , p. 78.

KовауаSні (H.). - Studies on the development of Diphyllobothrium mansoni ( $3 d$ report). Experimental studies on the mode of infection by the mature procercoïd. Taiwan Igakkai Zasshi, XXX, 1931, n 1, p. 3. (6th report). Taiwan Igakkai Zasshi, XXX, 1931, $\mathrm{n}^{\circ} 4$.

MUeller (J. F.). - The life history of Diphyllobothrium mansonoides Mueller, 1935. Amer. Jl. Trop. Med., XVIII, 1938, p. 41.

- Studies on Sparganum mansonoides and S. proliferum. Amer. Jl. Trop. Med., XVIII, 1938, p. 303.

- The hosts of Diphyllobothrium mansonoides. Proc. Helminthol. Soc. Washington, IV, 1937 , p. 68.

Nagoya (T.). - Route of migration of the orally fed Ligula mansoni in frog and mouse. Experim. Med., VIII, 1930, p. 29.

Faculté de Médecine de Hanoï. 